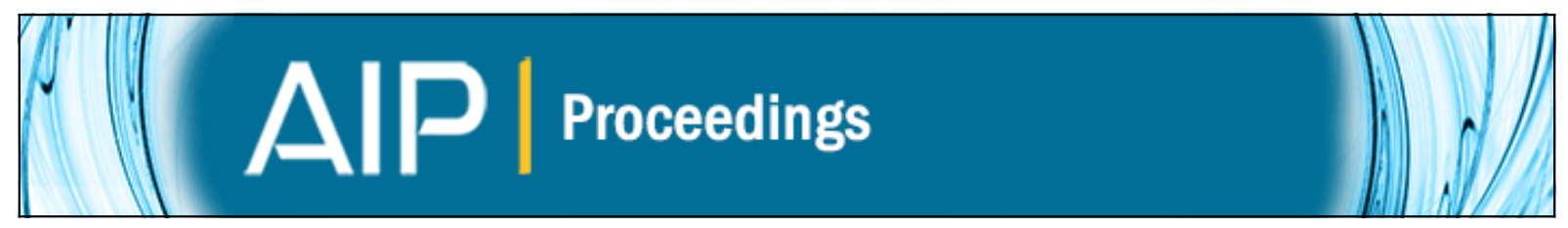

\title{
Overview of nonlinear kinetic instabilities
}

H. L. Berk

Citation: AIP Conference Proceedings 1478, 29 (2012); doi: 10.1063/1.4751638

View online: http://dx.doi.org/10.1063/1.4751638

View Table of Contents: http://scitation.aip.org/content/aip/proceeding/aipcp/1478?ver=pdfcov

Published by the AIP Publishing

Articles you may be interested in

Overview of beam instabilities

AIP Conf. Proc. 496, 3 (1999); 10.1063/1.1301870

Nonlinear modeling of kinetic plasma instabilities

Phys. Plasmas 6, 1822 (1999); 10.1063/1.873440

Critical nonlinear phenomena for kinetic instabilities near threshold

Phys. Plasmas 4, 1559 (1997); 10.1063/1.872286

Nonlinearly driven tearing instabilities by kinetic Alfvén waves

Phys. Fluids 30, 2457 (1987); 10.1063/1.866084

Nonlinear kinetic theory of a single helicity tearing instability

Phys. Fluids 27, 2043 (1984); 10.1063/1.864861 


\title{
Overview of Nonlinear Kinetic Instabilities
}

\author{
H.L.Berk \\ Institute for Fusion Studies, University of Texas at Austin
}

\begin{abstract}
The saturation of shear Alfvén-like waves by alpha particles is presented from the general viewpoint of determining the saturation mechanisms of basic waves in a plasma destabilized by a perturbing source of free energy. The formalism is reviewed and then followed by analyses of isolated mode saturation far from and close to marginal stability. The effect of multiple waves that are isolated or are overlapping is then discussed. The presentation is concluded with a discussion of a non-conventional quasilinear theory that covers both extreme cases as well as the intermediate regime between the extremes.
\end{abstract}

Keywords: energetic particles, Alfvén waves, marginal stability, wave-particle resonance, nuclear fusion

PACS: $89.30 . \mathrm{Jj}, 52.35 . \mathrm{Bj}$, 94.05.Pt

\section{INTRODUCTION}

The basic principle for achieving energy from controlled fusion is to confine a deuterium/tritium plasma at a temperature from $10-20 \mathrm{KeV}$, sufficiently well so that nuclear fusion occurs (through $D, T \rightarrow \alpha, n$ ) with a large gain above the energy input required to sustain this plasma. The neutrons, with a birth energy of $14 \mathrm{MeV}$, readily escape the plasma, and its energy will ultimately be converted to steam that will drive turbines generating electricity. Simultaneously, the electrically charged alpha particles, born at 3.5 $\mathrm{MeV}$, remain confined in the plasma. The alpha kinetic energy is intended to be transferred to the plasma background primarily through the drag slowing down process to the plasma's electrons [1]. This energy transfer balances energy loss processes, such as the energy flux from particle diffusion, to enable the plasma to remain at its high temperature. In principle a fusion machine, which for magnetic fusion is most likely to be a tokamak, need only be continually fed by the $D, T$ fuel with continual helium ash removal. In principle, no additional heating is needed, though auxiliary heating will be required for startup and for control of the fusion burn.

An essential question to address is whether the alpha particles, as shown confined in figure (1) in the JET machine, will be well confined and not be lost to the walls prematurely. Loss to the plasma facing walls depletes the heating source that maintains the plasma in its desired thermally hot state and unintentional alpha loss can cause excessive wall damage, if lost at $\mathrm{MeV}$ and 100' s of KeV energies. Thus, the confinement of alpha particles is an extremely important issue in the quest to attain commercial fusion energy power. 


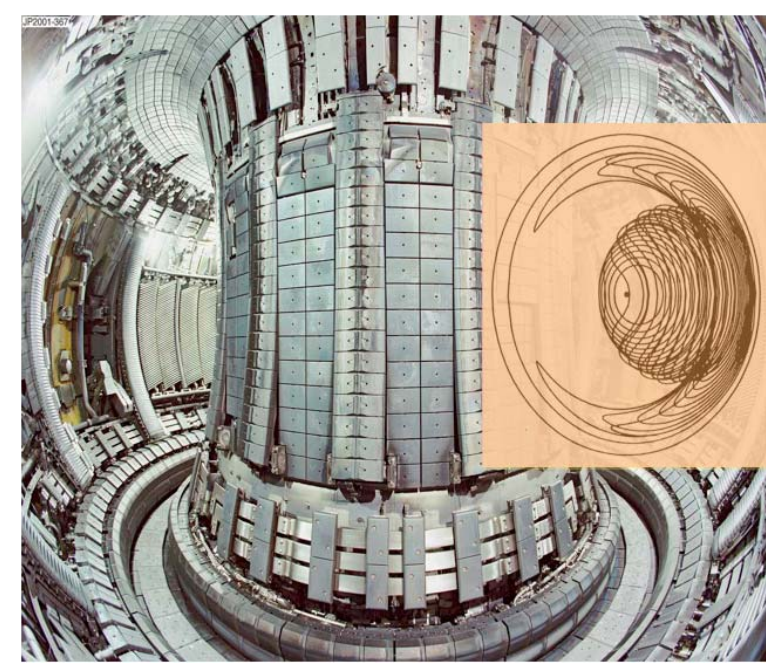

FIGURE 1. Schematic view of poloidal cross section of energetic particle orbits in the Joint European Tokamak (JET). The curves are projections of the particle trajectories onto the poloidal plane. The circular shaped curves are for passing particles while the crescent shaped (banana) orbits describe the trajectories of magnetically trapped particles.

\section{LINEAR INSTABILITY MECHANISM FROM ENERGETIC PARTICLES}

Alphas can be lost by magnetic field imperfections or by spontaneous generation of instability. Though we will not discuss the first topic, care must be taken to be sure that nearly all alpha particles would be confined in the absence of spontaneous wave perturbations, e.g. particle loss due to magnetic field ripple, arises because the toroidal magnetic fields are due to currents in a discrete magnet set rather than from a perfectly symmetric toroidal coil.

In this lecture we will discuss the issue of how waves saturate when they are excited spontaneously from instability. The understanding of this issue is very important in determining the margins for linear instability that can be tolerated in a tokamak. Indeed, it may be desirable to present a low level instability driven by alpha particles, so as to provide a diagnostic that helps determine the state of the burning plasma. Possible use of the wave 'free energy' of alpha particles to help improve fusion burn conditions is discussed by N. Fisch in this lecture series.

The basic instability mechanism for waves driven by alpha particles is the free energy available from the so-called Universal Instability Mechanism that arises from the spatial gradients in the alpha particle distribution function. This is the same mechanism that causes turbulent transport of plasma in conventional magnetically confined systems. It arises from the diamagnetic current flow that is associated with the spatial gradients of the particle distribution function.

To understand the basic reason for instability, first consider a slab geometry case where there is a uniform magnetic field in the $z$-direction. Take the plasma to be homogeneous in the $y$ and $z$ directions and spatial plasma confinement in the $x$-direction. Then the particle distribution, $F(\vec{r}, \vec{v}, t)$, will likewise be independent of $y$ and $z$ but de- 
pendent on $x$. As the particle distribution is stationary in time, it can be expressed in terms of the constants of motion, $F(\vec{r}, \vec{v}, t)=G\left(E, P_{y}, \mu\right)$, where $E=\vec{v}^{2} / 2 m+e \Phi(\vec{r}) / m$ is the particle energy, $P_{y}=v_{y}+\omega_{c} x$ is the canonical momentum in the $y$-direction, and the magnetic moment is,$\mu=v_{\perp}^{2} / 2 B$ (the three previous quantities are all per unit mass), $\omega_{c}=e B / m c$ is the cyclotron frequency, $e$ is the particle charge, $m$ is the particle mass and $c$ is the speed of light. If the distribution has a spatial scale large compared to the Larmor radius then approximately, $P_{y}=\Psi_{y} \equiv \omega_{c} x$. For simplicity we assume that the distribution does not depend on magnetic moment, $\mu$. Then we have:

$$
G\left(E, P_{y}\right)=G\left(E, \Psi_{y}+v_{y}\right) \approx G\left(E, \Psi_{y}\right)+\frac{\partial G\left(E, \Psi_{y}\right)}{\partial \Psi_{y}} v_{y} .
$$

Hence when we construct the plasma flow that arises from this particle distribution we find,

$$
v^{*}=\frac{1}{n_{p}} \int d^{3} v v_{y} G\left(E, P_{y}\right)=\frac{1}{n_{p}} \frac{\partial}{\partial \Psi_{y}} \int d^{3} v v_{y}^{2} G\left(E, \omega_{c}, \Psi_{y}\right)=\frac{1}{m n_{p}} \frac{\partial P}{\partial \Psi_{y}}=\frac{c}{e B n_{p}} \frac{\partial P}{\partial x},
$$

where $P=m \int d^{3} v v_{y}^{2} G\left(E, \omega_{c} x\right)$ is the kinetic pressure, and $n_{p}$ are the particle density and diamagnetic flow velocity in the y-direction respectively of the particle species being discussed. Thus, even for an otherwise isotropic distribution, there is a plasma flow, $v^{*}=\left(c / n_{p} e B\right) \partial P / \partial x$ in the negative $y$-direction (if $\partial P / \partial x$ decreases with increasing $x$ ) associated with the spatial gradient in the $x$-direction.

Now we note that the particles moving in the negative $y$-direction, with a speed $|v|$ that is in the range $0<|v|<\left|v^{*}\right|$ will, at a fixed $x$ position, be at speeds where the distribution is effectively inverted in energy (the distribution increases with decreasing speed). Because of this inversion, free energy can be spontaneously converted into wave energy. This energy release also causes motion across field lines. To see this, we consider a perturbed wave which is a function of $k_{y} y-\omega t$. As follows from the basic symmetry of the perturbation [2], the following relation between the change of energy $d E$ and change of canonical momentum $d P_{y}$ is satisfied as the wave evolves slowly compared to the frequency: $k_{y} d E / \omega=d P_{y}=\omega_{c} d x$ (it is important to note we are considering a resonance where $-\omega / k_{y}<-v^{*}$ is positive for a species with a positive charge). Thus when energy is lost from a positive charged particle, with a speed in the $y$-direction less than $v^{*}$, not only is energy lost but the particle moves spatially to larger $x$, i.e. down the spatial gradient. The lower the frequency the more pronounced is the cross field motion compared to the energy lost. For low frequency waves almost all the effect of losing energy is associated with the cross field motion of the particles.

Similar arguments apply to a toroidal plasma symmetric in the toroidal angle $\phi$, so that the canonical momentum associated with $P_{\phi}=R^{2} \dot{\phi}+e \Psi(r, \theta) / m c$ is a constant of motion, where $\Psi(r, \theta)$ is the poloidal magnetic flux with $B_{\perp}=-(1 / R) \partial \Psi(r, \theta) / \partial r$ the poloidal component of the magnetic field. Then an equilibrium distribution can be taken as a function of the energy $E$ and the canonical momentum $P_{\phi}=R^{2} \dot{\phi}+e \Psi(r, \theta) / m c$. Because of the toroidal symmetry of the equilibrium, the linear waves will be a function of $n \phi-\omega t$, where $n$ is an integer which causes the following relation between the change of particle energy, $d E$, and change of radial position, $d r ; n d E / \omega=d P_{\phi} \approx-\omega_{c \perp} R d r$ with 
$\omega_{c \perp}=e B_{\perp} / m c$ and $r$ the minor radius of the torus. Again it follows (when $n / \omega>0$ ) that as a particle gives up energy the particle moves outward in the $r$-direction. This cross field motion associated with low frequency waves, is the mechanism for anomalous diffusion.

As in the plasma slab case, one finds a diamagnetic rotation around the toroidal axis is at the diamagnetic rate $\omega^{*}=-\partial P / \partial r /\left(n_{p} m \omega_{c \perp} R\right)$ (note that $\omega^{*}>0$ if $\partial P / \partial r<0$, the usual case in a confined plasma) and any wave whose phase rotation is slower than the diamagnetic rotation frequency (i.e $0<\omega /\left(n \omega^{*}\right)<1$ ), is susceptible to a spontaneous release of free energy that causes radial diffusion. Especially, note that the ratio of the rotation rate of the energetic particles to the background species is the ratio of the mean energetic particle energy to the thermal energy of the background plasma species, which in a burning plasma is $\sim 100$. As a result considerably higher wave frequencies can cause radial loss of hot particles, than the frequencies that effect background plasma. The waves causing radial loss of background plasma are predominantly drift waves. However, the waves that could cause loss of energetic particles are higher frequency MHD-like waves, particularly those related to the shear Alfvén wave, which causes the magnetic field lines to bend while hardly changing the magnitude of the magnetic field.

\section{MARGINALLY UNSTABLE STATE}

In studying instability it is very important to consider conditions near marginal stability. The time scale for the classical slowing down of alpha particles is close to a second, whereas the time scale for instability growth, from $10^{-5}-10^{-4} \mathrm{sec}$, which is also the rough estimate for the time scale for relaxation of the nonlinear response. This discrepancy in time scales makes it likely that if a burning plasma is susceptible to alpha particle driven instability, that the system will relax in way to cause the system to hover near marginal instability. If there is enough phase space available for expansion of alpha particles without wall loss, the instabilities can remain benign in a state where there is continual Alfvén activity but without significant particle and energy loss. In that case the alpha particles will still continue to slow down within the plasma though perhaps further out from the region from the magnetic axis that would be predicted if instability is not accounted for. However, if the drive is too strong, the burn may be quenched due to the reduction of plasma heating due to loss of energetic alpha particles that no longer heat the background plasma and/or from the direct energetic particle flux on the plasma facing surfaces that causes wall deterioration. Thus detailed properties of the marginal stability state will affect whether a plasma burn can be sustained and hence it is important to understand this marginal state.

Though it may seem over-simplistic, the key to understanding saturation near marginal stability of Alfvénic modes in tokamaks, is to understand the saturation mechanism of instability of single modes for the bump-on-tail instability. The feature of the two problems have in common, is that a fundamental plasma excitation is present in absence of a destabilizing source (the electrostatic plasma wave in the case of the electrostatic bump-on-tail instability and the Toroidal Alfvén Wave (TAE) in the case of an Alfvénic wave in a burning plasma). The saturation mechanism in the two problems 
is then due to the following:

1. the presence of background dissipation mechanisms which will allow an inverted distribution of particles to remain stable until some critical gradient in the distribution is achieved;

2. the quenching of the instability drive by wave trapping [3] that flattens the distribution in the resonant region;

3. the presence of sources and sinks that allow a steady state balance to be achieved between the source, sink, and the relaxation mechanisms arising from the nonlinear response of the plasma.

The analysis to be discussed is not conventional quasilinear analysis. In the conventional quasilinear analysis a key implicit assumption is that enough different waves are excited such that the resonance width of the modes overlap. However, near marginal stability, where there are limited number of waves excited, the overlap condition is not necessarily attained. As a consequence, saturation is likely established due to the flattening of the distribution gradient due to wave trapping of particles in limited regions of phase space. Close enough to marginal stability, mode overlap is not likely to occur. There is then an important region to understand for how a plasma responds when a finite number of unstable modes are present whose resonance frequencies do not overlap. Then if mode overlap is achieved, the issues that arise are whether the system achieves steady excitations, a series of pulsations or perhaps even avalanches.

We begin with a discussion of the saturation due to trapping [4] of a single mode in the bump-on-tail electrostatic instability of a spatially homogeneous plasma. If a single mode is present it is readily shown that the particles satisfy the following (pendulum) equation:

$$
\frac{d^{2} \psi}{d t^{2}}+\omega_{b}^{2} \sin \psi=0
$$

where, $\omega_{b}^{2}=e k E_{0} / m, E_{0}$ is the normalized electric field amplitude and $m$ are the charge and mass of the particle and $\psi=k x-\omega t$ is the phase of the wave. The resonance condition for an unperturbed orbit is $\dot{\psi}=k v-\omega=0$, where $v=\dot{x}$. The field normalization is chosen so that $\omega_{b}$ is the trapping frequency of the most deeply trapped particles in the wave. Below we indicate why in the more general problem, Eq. (1) will still apply in terms of a trapping frequency, $\omega_{b}$, and a phase $\psi$, where their specific expressions depend on the details of the physical system being considered. Hence, as 3-D systems and the nonlinear electrostatic wave satisfy similar structural equations, much is gained by understanding the nonlinear properties of the bump-on-tail electrostatic problem.

Before a wave is excited in the bump-on-tail problem, the distribution of particles, with a weight $f_{a}$ just above the linear wave phase velocity (the red region of figure 2) has a larger weight than the weight of the distribution, $f_{b}$ below the resonant frequency (the blue region of the figure). If just a single mode is excited two phase space regions (i.e. the blue and red regions in fig. 2) will rotate clockwise in the trapping region at a shearing rotational rate, so that eventually the distribution weight of the two regions effectively mix, leading to an average of the two weights in the final mixed distribution. It is mostly the particles in the trapping region that can mix their weights, though due to non-adiabatic effects, there is mixing as well of particles in the region of phase space 


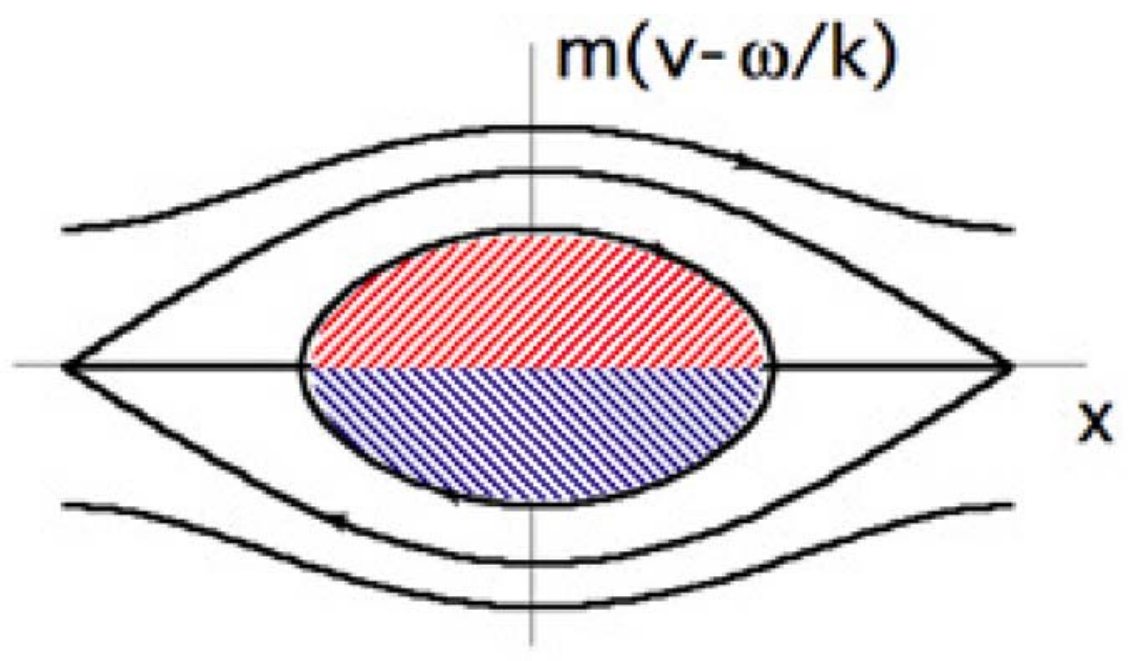

FIGURE 2. Phase space contours of constant energy in wave frame of a fixed amplitude standing wave.

slightly outside the separatrix. However, most of the particles outside the separatrix (passing particles) respond in an adiabatic manner and thus do not mix their distribution function, allowing the distribution to remain a constant function of their action variable during the entire evolution of the background wave. Hence the relaxation of the passing particle distribution is quite limited.

In fig. (3) we consider an energetic inverted distribution function as it increases with increasing energy. There is then the potential for spontaneously release of free energy from a region of action space $I$ at a fixed frequency $\omega \approx \Omega(I)=k v . \Omega(I)$ is the frequency of the particle's action variable and $k v$ is the particular form of this action variable that applies to the bump-on-tail problem. As a result of the mixing of the distribution within the separatrix, shown in fig. (2), the mean value for the distribution flattens in the region $\Omega(I) \sim \omega$ as is shown in fig. (3). Outside the separatrix region, the distribution is essentially the same as it would be without any wave excitation while there is a relatively sharp transition region around the separatrix.

When several modes are excited, one can have extreme situations, that can be compared. In fig. (4a), the multiple discrete waves do not overlap, so that the distribution only mixes within the separatrix of each isolated resonance while between resonances, the distribution is hardly changed. In contrast, in fig.4(b) as a consequence of the multiple resonance overlap, the particle mixing is no longer confined within the separatrix of a single resonance. As a result the distribution mixes over a much larger region of phase space. This much larger mixing region clearly reduces the energy of the resonant particles, far more than in the non-overlapping case. Hence, to the extent energy is conserved between energetic particles and waves, there is a much larger enhancement of the wave amplitudes in the overlapping case compared to the mixing case. Hence, fig.(4a) depicts relatively benign relaxation as there is hardly any transport of resonant particles, while fig.(4b) depicts pronounced nonlocal particle transport and even an amplification of the wave amplitude. These two examples give two possible extreme outcomes during the evolution of a marginally unstable state. 


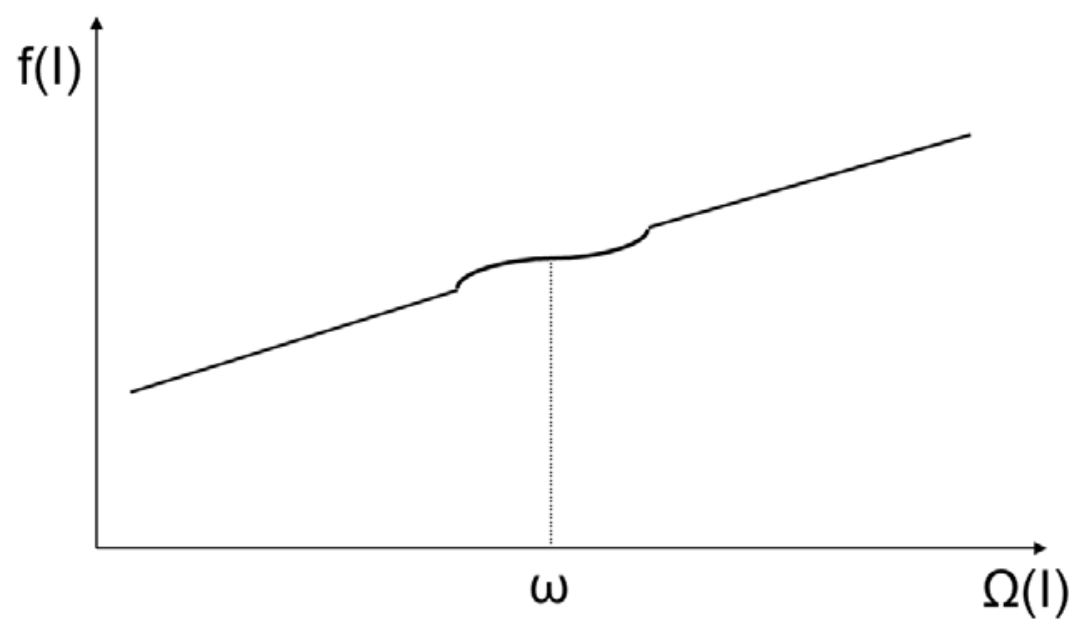

FIGURE 3. Flattening of distribution due to mixing within the separatrix region.
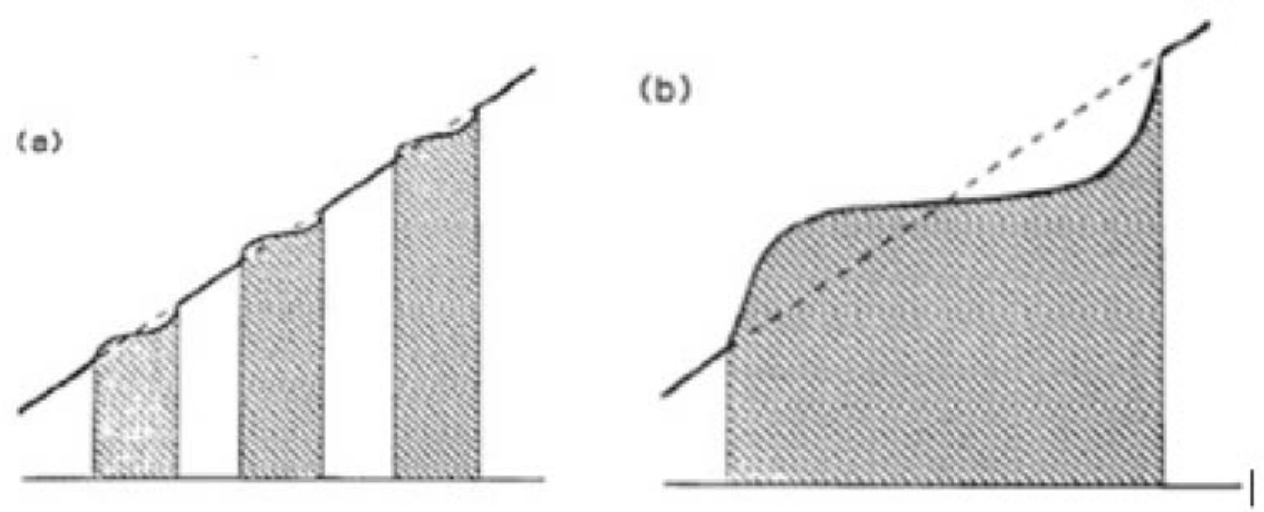

FIGURE 4. (a) local flattening when waves do not overlap; (b) global flattening arising when waves overlap.

\section{FORMALISM FOR NONLINEAR WEAKLY DRIVEN KINETIC INSTABILITY}

In this section we review the formalism that allows us to use the simple 1-D picture that is based on the electrostatic bump-on-tail problem, for problems in more complicated geometry, such as description of phenomena in an axisymmetric tokamak. We note that in axisymmetric geometry the unperturbed orbits exactly conserve two quantities, the particle canonical momentum $P_{\phi}$, the particle energy $E$ and a third approximately conserved invariant (to within exponential accuracy) the magnetic moment $\mu$. Three angles and frequencies associated with the motion of charged particles in a tokamak 
are, the toroidal angle $\phi$ matched with its mean angular frequency $\omega_{\phi}\left(E, P_{\phi}, \mu\right)$, the poloidal angle $\theta$ with a mean angular frequency $\omega_{\theta}\left(E, P_{\phi}, \mu\right)$, and the gyro-phase angle $\psi$ with its mean gyrofrequency $\omega_{\psi}\left(E, P_{\phi}, \mu\right)$. These six coordinates determine the six dimensional phase space positions of any orbit in the tokamak. The resonance condition can be written as: $\omega-n \omega_{\phi}\left(E, P_{\phi}, \mu\right)-l \omega_{\theta}\left(E, P_{\phi}, \mu\right)-s \omega_{\psi}\left(E, P_{\phi}, \mu\right)=0$, where the quantities, $l, n$ and $s$ are integers with $\mathrm{s}=0$, which is the case for resonances associated with low-frequency modes (less than the cyclotron frequency).

The unperturbed particle orbits are usually integrable, which then allows the coordinates to be transformed into three action variables, $\vec{J} \equiv\left(J_{1}, J_{2}, J_{3}\right)$ and to their three respective conjugate angles, $\vec{\chi} \equiv\left(\chi_{1}, \chi_{2}, \chi_{3}\right)$. Recall that these conjugate angles have the properties that for unperturbed motion, $\dot{\vec{\chi}}=$ constant. It is this property that allows the simple one dimensional bump-on-tail problem to be the basic paradigm for describing a complex three dimensional tokamak problem and indeed, for many other physical problems. In a tokamak the actions can be chosen as $J_{3}=\mu, J_{1}=P_{\phi}$ while the remaining action $J_{2}$ is related to the energy by $d J_{2}=d E / \omega_{\theta}$ at constant $J_{3}$ and $J_{1}$.

Now consider an electromagnetic toroidal perturbation applied to the system (for succinctness of presentation, a gauge is chosen where the electrostatic potential vanishes). The dynamics of the particle motion is determined by a Hamiltonian, that is a function of momentum $\vec{p}$, position $\vec{r}$, and time $\mathrm{t}$, taken to be of the form,

$$
H(\vec{p}, \vec{r}, t)=H_{0}(\vec{p}, \vec{r})+\frac{e \vec{A}_{0}(\vec{r}) \cdot \vec{v}(\vec{p}, \vec{r})}{2 c}[\exp i(n \phi-\omega t)+c . c]
$$

where $\vec{A}_{0}(\vec{r}) \cos (n \phi-\omega t)$ is the perturbed vector potential and $H_{0}(\vec{p}, \vec{r})$ and $\frac{e}{c} \vec{A}_{0}(\vec{r})$. $\vec{v}(\vec{p}, \vec{r})$ are independent of the toroidal angle $\phi$.

In terms of action angle variables, this Hamiltonian can be written as,

$$
H(\vec{p}, \vec{r}, t)=H_{0}(\vec{J})+\frac{1}{2} \sum_{\vec{s}}<\frac{e}{c} \vec{A}_{0}(\vec{r}) \cdot \vec{v}(\vec{J})>_{\vec{s}}[\exp i(\vec{s} \cdot \vec{\chi}-\omega t)+c . c]
$$

where,

$<\frac{e}{c} \vec{A}_{0} \cdot \vec{v}(\vec{J})>_{\vec{s}}=\lim _{T \rightarrow \infty} \frac{1}{T} \int_{0}^{T} d t \frac{e \vec{A}_{0} \cdot \vec{v}(\vec{r}(t)) \exp [i n \phi(t)]}{2 c} \exp \left[-i l \chi_{1}(t)-i n \chi_{2}(t)-i s \chi_{3}(t)\right]$

where the time dependence in the integrand is taken over the unperturbed orbits of a particle.

Also, note that for the unperturbed system $\dot{\vec{\chi}}(t)=\omega(\vec{J})=$ const. If the amplitude is small enough, the response for the non-resonant perturbations can be taken as linear with $\exp i(\vec{s} \cdot \vec{\chi}(t)-\omega t)$ considered to be a rapidly varying function of time. However, for resonant, indices $\vec{s}_{0}$, the response in time has to be treated nonlinearly. We define resonant surfaces in phase space for $\vec{J}$ which satisfy, $\vec{s}_{0} \cdot \vec{\chi}(t)-\omega t=0$. For the particles that nearly satisfy this resonance, the response readily becomes nonlinear even for a relatively small amplitude wave. The nonlinear solution to the resonant particle response is obtained by neglecting the non-resonant terms in Eq.(3). As a result the near resonant particle dynamics is well described by a Hamiltonian that consists of the unperturbed Hamiltonian 
plus only those terms in Eq. (3) for which $\vec{s}=\vec{s}_{0}$ where $\Omega_{\vec{s}_{0}}\left(J_{0}\right) \equiv n \omega_{\phi}\left(J_{0}\right)+l \omega_{\theta}\left(J_{0}\right)=\omega$ and we have taken the low frequency case where $s=0$, which will apply to the remaining discussion. With this truncation and expanding $H(\vec{J})$ to second order in $\vec{J}-\vec{J}_{0}$ the governing resonant particle reduced Hamiltonian, $H_{r d c}$, is found to be given by,

$$
H_{r d c}=\left.\frac{\left(J-J_{0} / n\right)^{2}}{2} \frac{\partial \Omega_{0}\left(\vec{J}_{0}\right)}{\partial J}\right|_{J_{2}^{\prime}, J_{3}}+\frac{1}{2}\left(<\frac{e}{c} \vec{A}_{0} \cdot \vec{v}(\vec{J})>_{\vec{s}_{0}} \exp (i \chi)+c . c\right)
$$

with $\chi=l \chi_{1}+n \chi_{2}-\omega t, J=J_{1} / n$ and the constants of motion are $J_{2}^{\prime}=J_{2}-l J_{1} / n$ and $J_{3}$ as the reduced Hamiltonian is independent of the phase angles $\chi_{2}$ and $\chi_{3}$ conjugate to $J_{2}^{\prime}$ and $J_{3}$. It follows from the reduced Hamiltonian equations of motion for $J$ and $\chi$ that the governing equation for the phase of the resonant particle response is given by the same pendulum equation as Eq. (1), but now with a more general expression for the wave trapping frequency given by,

$$
\omega_{b}^{2}=<\frac{e}{c} \vec{A} \cdot \vec{v}(\vec{J})>\left._{\vec{s}_{0}} \frac{\left.\partial \Omega_{\vec{s}_{0}}\left(\vec{J}, J_{2}^{\prime}, J_{3}\right)\right)}{\partial J}\right|_{J_{2}^{\prime}, J_{3}}
$$

In addition it follows that the kinetic equation for the resonant particles can be expressed in terms of a distribution function, $f\left(\Omega, \vec{\chi} ; J_{2}^{\prime}, J_{3}\right)$, with $\Omega=$ $\omega+\left(J-J_{10} / n\right) \partial \Omega_{\vec{s}_{0}}\left(J_{10}\right) / \partial J_{1}$ that has the same structure as the Vlasov equation for the bump-on-tail problem. The resonant particle distribution function, $f_{r s}$ (the rs subscript will usually be suppressed), satisfies the equation:

$$
\left[\frac{\partial}{\partial t}+\Omega \frac{\partial}{\partial \chi}+\omega_{b}^{2} \sin \chi \frac{\partial}{\partial \Omega}\right] f(\Omega, \chi, t)=v_{e f f}^{3} \frac{\partial^{2}\left(f(\omega, \chi, t)-f_{S}(\Omega)\right)}{\partial \Omega^{2}}
$$

where on the RHS we have added to the Vlasov equation a diffusive relaxation term and a particle source term, which is the one containing $f_{S}(\Omega)$.

The diffusive relaxation term can be related to such physical stochastic processes, as collisions, heating, etc, that is always part of a physical system.

Observe that the kinetic equation given by Eq.(7) is just as applicable to resonant particles in the bump-on-tail problem as waves in a tokamak (indeed to any system where equilibrium orbits are integrable). The only difference in the two problems is that there is only one region of resonant particles in the bump-on-tail problem, while in the tokamak problem there are two dimensional surfaces for a given $\omega$ with indices $\vec{s}_{0}$ that satisfy the resonance condition, that need to be integrated over.

In addition we need to describe the wave response. We start from Maxwell's equations where the non-resonant linearly perturbed current is taken to be the reactive part of the plasma current response. In addition we add an extrinsic dissipative part that comes from collisional effects and/or stabilizing resonant particle effects. An additional assumption used is that the spatial shape of the wave fields remain unchanged during the evolution of the wave, while the temporal response of the wave changes. Such an assumption can be justified when frequencies are close to the eigenfrequencies of the background plasma.

In linear theory, the reactive plasma current, $\vec{j}_{r c}(\vec{r}, t)$, which is related to the perturbed electric field by a non-local conductivity tensor, $\overrightarrow{\vec{\sigma}}_{r c}\left(t-t^{\prime}, \vec{r}, \vec{r}^{\prime}\right)$, that depends on space 
and time and produces a reactive current of the form,

$$
\vec{j}_{r c}(\vec{r}, t)=\int_{-\infty}^{t} \overrightarrow{\vec{\sigma}}\left(t-t^{\prime}, \vec{r}, \vec{r}^{\prime}\right) \cdot \vec{E}\left(\vec{r}^{\prime}, t^{\prime}\right)=-c^{-1} \int_{-\infty}^{t} \overrightarrow{\vec{\sigma}}\left(t-t^{\prime}, \vec{r}, \vec{r}^{\prime}\right) \cdot \frac{\partial \vec{A}\left(\vec{r}^{\prime}, t^{\prime}\right)}{\partial t^{\prime}} .
$$

There is also a current due to extrinsic dissipation, which is simply modeled as $\vec{j}_{d s}(\vec{r}, t)=$ $\sigma_{e x} \vec{E}(\vec{r}, t)$. Finally, there is the current arising from the resonant regions of the plasma, $\vec{j}_{r s}(\vec{r}, t)$. Only the resonant currents are treated as nonlinear and the distribution function in the resonant phase space region is taken to satisfy a reduced Vlasov equation given by Eq. (7). The vector potential is taken to be of the form, $\vec{A}(\vec{r}, t)=$ $C(t) \vec{a}\left(\vec{r} ; \omega_{0}\right) \exp \left(-i \omega_{0} t\right) / 2+c . c$ where $C(t)$ is a complex amplitude with the assumed property that $d C(t) / d t /(\omega(t) C(t)) \ll 1$ and $\vec{a}\left(\vec{r} ; \omega_{0}\right)$ is the linear eigenfunction of the wave for eigenvalue $\omega=\omega_{0}$. Substituting the currents into Maxwell equations leads to the wave equation,

$$
\begin{aligned}
& C(t)\left[\vec{\nabla} \times \vec{\nabla} \times \vec{a}(\vec{r})-\frac{\omega_{0}^{2}}{c^{2}} \vec{a}(\vec{r})-\frac{4 \pi \omega_{0}}{c^{2}} \int d^{3} \overrightarrow{r^{\prime}} \Sigma\left(\omega_{0}, \vec{r}, \vec{r}^{\prime}\right) \cdot \vec{a}\left(\vec{r}^{\prime}\right)\right] \\
& -i \frac{4 \pi \omega_{0}}{c^{2}} \sigma_{e x} \vec{a}(\vec{r}) C(t)+i \frac{d C(t)}{d t} \frac{\partial}{\partial \omega_{0}} \\
& {\left[\vec{\nabla} \times \vec{\nabla} \times \vec{a}(\vec{r})-\frac{\omega_{0}^{2}}{c^{2}} \vec{a}(\vec{r})-\frac{4 \pi \omega_{0}}{c^{2}} \int d^{3} \vec{r}^{\prime} \Sigma\left(\omega_{0}, \vec{r}, \vec{r}^{\prime}\right) \vec{a}\left(\vec{r}^{\prime}\right)\right]=\frac{4 \pi}{c} \vec{j}_{r s}(t) \exp \left(i \omega_{0} t\right)}
\end{aligned}
$$

where $-i \int d^{3} \overrightarrow{r^{\prime}} \Sigma\left(\omega_{0}, \vec{r}, \vec{r}^{\prime}\right)$, the Laplace transform of time of the spatially non-local conductivity tensor with the resonance response omitted, which is a Hermitian selfadjoint operator. Now take the dot product of this function with $\vec{a}^{*}\left(\vec{r} ; \omega_{0}\right)$, integrate over all space, neglecting highly oscillatory terms to obtain for the wave amplitude evolution:

$$
\begin{aligned}
& G\left(\omega_{0}\right) C(t)+i \frac{4 \pi \omega_{0}}{c^{2}} \int d^{3} \vec{r} \sigma_{e x}|\vec{a}(\vec{r})|^{2} C(t)+i \frac{\partial G\left(\omega_{0}\right)}{\partial \omega} \frac{d C(t)}{d t} \\
& =-\frac{4 \pi}{c} C(t) \int d^{3} \vec{r} \vec{j}_{r s} \cdot \vec{a}^{*}(\vec{r}) \exp \left(i \omega_{0} t\right)
\end{aligned}
$$

where

$$
\begin{aligned}
G(\omega) & =\iint \frac{d^{3} \vec{r} d^{3} \vec{r}^{\prime}}{4 \pi}\left\{-\delta\left(\vec{r}-\vec{r}^{\prime}\right)\left[\nabla^{\prime} \times \vec{a}^{*}\left(\vec{r}^{\prime}\right) \cdot \nabla \times \vec{a}(\vec{r})+\frac{\omega^{2}}{c^{2}} \delta\left(\vec{r}-\vec{r}^{\prime}\right) \vec{a}^{*}\left(\vec{r}^{\prime}\right) \cdot \vec{a}(\vec{r})\right]\right. \\
& \left.-\frac{4 \pi \omega}{c} \vec{a}^{*}\left(\vec{r}^{\prime}\right) \cdot \Sigma_{r s}\left(\omega, \vec{r}, \vec{r}^{\prime}\right) \cdot \vec{a}\left(\vec{r}^{\prime}\right)\right\}
\end{aligned}
$$

Note that $\vec{a}(\vec{r})$ is eigenfunction obtained by solving the first bracketed term on the LHS of Eq. (9) set to zero, from which one finds the eigenvalue $\omega=\omega_{0}$. For the eigenvalue, $\omega=\omega_{0}$ one can show that $G\left(\omega_{0}\right)=0$ and this expression can be viewed as the linear dispersion relation for the basic undamped wave in a toroidal plasma. Hence, the wave equation for determining the wave complex amplitude $C(t)$ is

$$
\frac{\partial G\left(\omega_{0}\right)}{\partial \omega} \frac{d C(t)}{d t}+\frac{4 \pi \omega_{0}}{c^{2}} \int d^{3} \vec{r} \sigma_{e x}|\vec{a}(\vec{r})|^{2} C(t)=i \frac{4 \pi}{c} C(t) \int d^{3} \vec{r} \vec{j}_{r s} \cdot \vec{a}^{*}(\vec{r}) \exp \left(i \omega_{0} t\right)
$$


Further note that by multiplying Eq. (12) by $C^{*}(t)$ and the complex conjugate of Eq. (12) by $C(t)$ and then adding the two terms, we obtain the equation,

$$
\begin{aligned}
\frac{\partial G\left(\omega_{0}\right)}{\partial \omega} \frac{d\left(C^{*}(t) C(t)\right)}{d t} & =\frac{4 \pi}{c} C^{*}(t) C(t)\left(i \int d^{3} \vec{r} \vec{j}_{r s} \cdot \vec{a}^{*}(\vec{r}) \exp \left(i \omega_{0} t\right)+c . c\right) \\
& -\frac{8 \pi \omega_{0}}{c^{2}} \int d^{3} \vec{r} \sigma_{e x}|\vec{a}(\vec{r})|^{2}|C(t)|^{2}
\end{aligned}
$$

In addition one can show that $\frac{C^{*}(t) C(t)}{4} \partial G\left(\omega_{0}\right) / \partial \omega, \omega_{0} \frac{C^{*}(t) C(t)}{4} \partial G\left(\omega_{0}\right) / \partial \omega$ and $n \frac{C^{*}(t) C(t)}{4} \partial G\left(\omega_{0}\right) / \partial \omega$ are respectively the wave action, wave energy and wave angular momentum along the axis of the axisymmetric torus. One may then interpret Eq.(13) as the time rate of change of wave action (i.e. rate of change of the number of wave photons) is due to the interaction of the resonant particle currents and dissipative currents with the perturbed fields.

In solving for the wave equation, we need to solve for the resonant current response,

$$
\begin{aligned}
\int d^{3} \vec{r}_{r s} \cdot \vec{a}^{*}(\vec{r}) & =e \int d^{3} \vec{r} d^{3} \vec{v} f_{r s} \vec{v} \cdot \vec{a}^{*}(\vec{r}) \\
& =(2 \pi)^{2} \int d J_{2}^{\prime} d J_{3} \frac{\partial J_{1}}{\partial \Omega} \sum_{\vec{s}_{0}} \int d \chi d \Omega f_{r s}\left(\vec{J}, \vec{\chi} ; J_{2}^{\prime}, J_{3}\right)<e \vec{v} \cdot \vec{a}^{*}(\vec{r})>_{\vec{s}_{0}}
\end{aligned}
$$

where $f_{r s}\left(\vec{J}, \vec{\chi} ; J_{2}^{\prime}, J_{3}\right)$ is solution for the resonant particle Vlasov equation given by Eq. (7). Thus Eqs. (7), (13) and (14) correspond to a closed set of equations.

These equations describe the nonlinear response of resonant particles that produce weakly driven instabilities. The basic difference between the simple bump-on-tail electrostatic problem and the three dimensional problem is that additional integrations in the $J_{2}^{\prime}$ and $J_{3}$ and summation of resonances, denoted by $\vec{s}_{0}$, are required for the 3 -d problem while for the electrostatic 1-d problem these integrations are absent. Otherwise the basic structures of the problems are identical. Thus by in large we can limit our discussion to the simple properties of the 1-d problem, and achieve insight needed to understand the nonlinear response of alpha particles driving TAE modes in a burning plasma.

\section{SATURATION LEVELS}

\section{a. Saturation without extrinsic damping, relaxation or particle sources}

We now discuss the saturation levels in terms of the trapping frequency, $\omega_{b}$ for the bump-on-tail instability. First consider the case where a single mode is excited, and there is an energetic particle drive that produces a growth rate $\gamma_{L}$, when there is no background dissipation present. Then in the evolution of the mode, the total momentum must be conserved. Hence, in the final saturated state, the increase of the final wave momentum (this includes the electromagnetic field momentum plus the increased momentum of the non-resonant particles oscillating coherently with the wave) is attained through the loss of momentum of the resonant particles. The resonant distribution relaxes from a 
finite slope to a nearly zero slope in the trapping region, as shown in fig.(3), as well as producing a distortion of the distribution of passing particles close to the resonance.

The final state of the system also depends somewhat on the dynamical path to saturation. Two contrasting scenarios are readily described analytically by using that the final distribution of trapped particles to be the average of the phase space regions of the unperturbed system that transition to the trapping region as the wave grows to saturation. Different dynamical paths to saturation still give some uncertainty in the theoretical prediction as to what level of saturation will be reached. At one extreme we can assume that the fields evolve so slowly that the particles remain adiabatic, so that the distribution remains a function of the action during the evolution, though with the distribution mixing in the trapping region. Due to this mixing, a prediction of a saturation level, $\omega_{b}=2.9 \gamma_{L}$ is inferred. The other extreme is to assume that the final saturated field amplitude is established suddenly as an impulse, whereupon the particles mix at constant field amplitude. Then total momentum conservation leads to a prediction that $\omega_{b}=3.5 \gamma_{L}$. A numerical simulation of the problem [5] gives as the final trapping frequency, $\omega_{b}=3.2 \gamma_{L}$, a result that lies between these two estimates. Thus we see that particle trapping leads to a saturation level that scales with the linear growth rate. The flattened region shown in fig.(3) has a width $\Delta \Omega \approx 3 \gamma_{L}$.

\section{b. Saturation with a source and sink with weak extrinsic damping}

Now consider the case where the distribution function is established with a source and sink that is well above the marginal stability of a discrete mode. In that case the instability growth rate $\gamma_{L}$ in absence of damping is substantially above the damping rate $\gamma_{d}$ that would arise in absence of the drive. For a steady state solution, the time derivative terms of Eqs. (7) and (13) are absent. As the result justifies a priori, we assume that the solution has the character that $\omega_{b}>>v_{e f f}$. Then to obtain an analytic result, we develop a perturbation procedure for the $p^{\text {th }}$ iterant based on the following iteration,

$$
\Omega \frac{\partial f_{p}}{\partial \psi}-\omega_{b}^{2} \sin \psi \frac{\partial f_{p}}{\partial \Omega}=v_{e f f}^{3} \frac{\partial^{2} f_{p-1}}{\partial \Omega^{2}}
$$

with a boundary condition that $\frac{\partial f_{0}}{\partial \Omega} \longrightarrow \frac{\partial F(\Omega)}{\partial \Omega}|\Omega| \gg \omega_{b}$, where $F(\Omega)$ is the energetic particle distribution established by the classical sources and sinks without any waves present.

Here we discuss how to use dimensional analysis in this iteration to produce the appropriate scaling for the saturated solution. To lowest order, $p=1$, Eq. (15) gives: $\Omega \frac{\partial f_{0}}{\partial \psi}-\omega_{b}^{2} \sin \psi \frac{\partial f_{0}}{\partial \Omega}=0$. By balancing the two terms of the lowest order equation, $\partial f_{0} / \partial \psi \sim f_{0} \sim \omega_{b}^{2} f_{0} / \Omega^{2}$, we find that the range of sharp change of $f_{0}$ with respect to $\Omega$ occurs where $\Omega \approx \omega_{b}$, while the need to have the slope, $\partial f_{0} / \partial \Omega$, in the inner region be comparable to the slope in the outer region, lead to : $\partial f_{0} / \partial \Omega \sim f_{0} / \omega_{b} \sim \partial F / \partial \Omega$. Consequently, $f_{0} \approx \omega_{b} \partial F / \partial \Omega$. Similar scaling considerations then give to next order, $f_{1} \approx\left(v_{e f f} / \omega_{b}\right)^{3} f_{0} \approx\left(v_{e f f}^{3} / \omega_{b}^{2}\right) \partial F(\Omega) / \partial \Omega$. 
Now to estimate the saturation level we use that the nonlinear power transfer from the energetic particles to the plasma, as given by Eq. (13), with the aid of Eq. (14), has to be reduced from the one deduced from the linear theory expression by a factor of $\gamma_{d} / \gamma_{L}$. Thus we find,

$$
\frac{\gamma_{d}}{\gamma_{L}}=\frac{\int d J_{2}^{\prime} d J_{3}\left(\frac{\partial J_{1}}{\partial \Omega}\right)^{2} \int d \chi d \Omega\left[\left(f_{0}(\Omega)+f_{1}(\Omega, \chi)\right)\left|\omega_{b \vec{s}}^{2}(\vec{J})\right| e^{i \chi}+c . c .\right]}{2 \int d J_{2}^{\prime} d J_{3}\left(\frac{\partial J_{1}}{\partial \Omega}\right)^{2} \sum_{\vec{s}} \int d \chi \frac{\partial F}{\partial \Omega} \delta(\Omega) d \Omega\left|\omega_{b \vec{s}}^{2}(\vec{J})\right|^{2}}
$$

The right hand side of this equation is ratio of nonlinear to linear power release of the energetic particles. Also note that because the $f_{0}$ term in the numerator is independent of $\Omega$, this term vanishes upon $\chi$ integration. If we then substitute the estimate $f_{1} \sim$ $\left(v_{e f f}^{3} / \omega_{b}^{2}\right) \partial F(\Omega) / \partial \Omega$, and use that the range of the $\Omega$ integration in the numerator is $\omega_{b}$, we find the scaling for $\omega_{b}$ to be.

$$
\omega_{b} \sim v_{d}\left(\frac{\gamma_{L}}{\gamma_{d}}\right)^{1 / 3}
$$

For the bump-on-tail instability, the quantitative calculation gives $\omega_{b}=1.2 v_{d}\left(\frac{\gamma_{d}}{\gamma_{L}}\right)^{1 / 3}$ [4].

Closer to marginal stability, when $\gamma_{L}-\gamma_{d} \ll \gamma_{L}$, the saturated trapping frequency has been shown to take the form found in [6].

$$
\omega_{b}=1.4\left(1-\frac{\gamma_{L}}{\gamma_{d}}\right)^{1 / 4} v_{e f f}
$$

An understanding of how this scaling comes about from simple balancing arguments similar to what we have just discussed, is given in [7].

To predict the saturation level in between the two limiting regimes $\gamma_{L} \gg \gamma_{d}$ and $\gamma_{L}-\gamma_{d} \ll \gamma_{L}$, an interpolation formula has been used. The results of this interpolation compares favorably with the results from simulations as is shown in figure (5). (The figure on the left is for the bump-on-tail problem simulated by Petviashvili [8] and the figure on the right is for the TAE mode simulated by Y. Chen [7]).

The scaling formulas we have just presented in this sub-section then allows one to estimate the mode width $\Delta \Omega \sim \omega_{b}$ in terms of the growth rate, $\gamma_{L}$, the damping rate $\gamma_{d}$ and the diffusive relaxation rate $v_{e f f}$. This estimate is very important for establishing saturation level in the quasilinear formalism that will be discussed below in the section on quasilinear theory.

\section{c. Pulsation Scenario}

The previous section produced solutions that give a steady state saturated response where there was a balance between a source input, an instability drive and a basic diffusive relaxation mechanism. However, it is also possible that the wave response will not be steady, even when there is just a single resonance excitation. Then one has to consider the possibility of a non-steady response. An indication of this possibility is the prediction from Eq. (16) that shows that the saturated wave amplitude becomes 
Bump on tail

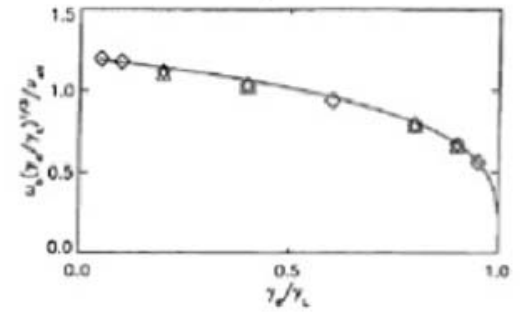

TAE

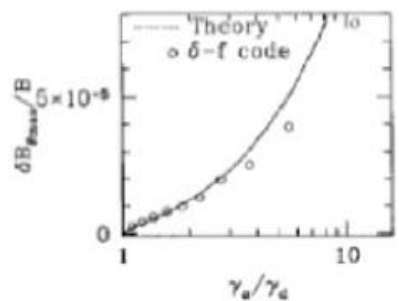

FIGURE 5. Comparison of the normalized saturation levels determined from simulation and interpolated analytic formulae. The figure on the left is for the bump-on-tail instability while that on the right is for the TAE instability as a function of $\gamma_{d} / \gamma_{L}$. The solid curve is the interpolated calculation, while the discrete points are results of the simulation. The triangular and square points in the left figure are for different values of $\gamma_{L}$.

arbitrarily small as $v_{\text {eff }}$ goes to zero. In that case the saturated distribution would hardly be distorted upon saturation, and hence one might expect the instability growth to continue. Indeed, it was shown in reference [9] that the steady solution is unstable. What should then happen is that the instability continues to grow essentially at the linear growth rate, until the trapping frequency of the mode becomes comparable to the linear growth rate. Then the nonlinear response that is attempting to produce over-turning of the trapped particles within the separatrix, has enough time compared to the linear growth time to complete this task. Then the distribution function in the resonance region $\Delta \Omega \sim \omega_{b}$, flattens causing a depletion of the drive for the mode, thereby enabling mode stabilization. We call this saturation level the natural saturation amplitude. If $v_{e f f} \ll \gamma_{d}$, the mode amplitude will damp on a time scale $t_{d m p} \sim 1 / \gamma_{d}$, while the distribution is flattened over a region centered about the resonant frequency, for a width in phase space $\Delta \Omega \sim \omega_{b} \sim \gamma_{L}$.

The restoration of the slope in the distribution to a value comparable to the slope of the surrounding ambient distribution function is brought about by a normalized phase space diffusion coefficient given by $v_{e f f}^{3} \sim v_{c l} \omega^{2}$ where $v_{c l}$ is the large angle scattering rate. If $v_{e f f} \ll \gamma_{d}$, standard diffusion arguments then produces the following scaling for the restoration time, $t_{r s t} \sim \gamma_{L}^{2} / v_{\text {eff }}^{3}$, in a region $\Delta \Omega \sim \gamma_{L}$, that goes from a flattened phase space region to the slope of the fully developed instability. When restoration is near completion, the slope of the distribution becomes large enough to produce a linear instability drive that can overcome the background damping, and the linear instability is excited again. Thus we expect a saw-tooth like response, with a sharp onset of instability at a rate $\gamma_{L}$, a slower decay of the excitation at a rate $\gamma_{d}$ followed by longer quiescent time $t_{r s t}$.

It is worth noting that when $v_{e f f}>\gamma_{L}$ (where the saturation level is in steady state with the saturation amplitude $\left.\omega_{b} \sim v_{e f f}\left(\gamma_{L} / \gamma_{d}\right)^{1 / 3}\right)$ the saturation level is always intrinsically larger than the natural saturation level $\omega_{b} \sim \gamma_{L}-\gamma_{d}$ (applicable when $\gamma_{d}$ is not too close to $\gamma_{L}$ ) of the pulsation state. Thus diffusion from the ambient distribution, which is established by an external source and classical relaxation processes, pumps up the 
saturation level when $v_{e f f}>\gamma_{L}$. Roughly then, the saturation level, independent of numerical coefficients of order unity and taking into account Eqs. (16) and (17) can be written in the following form,

$$
\omega_{b} \sim v_{e f f}\left(\left(1-\frac{\gamma_{d}}{\gamma_{L}}\right)^{1 / 4}+\left(\frac{\gamma_{L}}{\gamma_{d}}-1\right)^{1 / 3}\right)+\left(\gamma_{L}-\gamma_{d}\right)
$$

An unexpected response occurs when $v_{e f f} /\left(\gamma_{L}-\gamma_{d}\right)<1$, where oscillations and explosions can occur. Indeed, the explosive state turns out to be a precursor to rapid frequency chirping due to the generation of generalized BGK nonlinear wave [10]. This scenario is the topic of accompanying lectures by M.Lilley. The saturated level of the chirping structure is found to be $\omega_{b} \sim 0.5 \omega_{L}$. This is the same scaling as the case with $\gamma_{d}=0$, but with a numerical factor that is 6 times lower.

\section{d. Mode overlap}

When there are several modes present, the modes will oscillate independently of each other when the mode width, $\sim \omega_{b}$ is much less than the mode frequency separation $\Delta \omega$. However, with a source present, the overall level of the energetic particle population will continue to rise, creating a larger slope in the distribution. In figure (6) the dashed line represents the critical slope needed to achieve marginal stability. Suppose initially we take the distribution's slope, to be less than the critical slope. Then the background dissipation is large enough to overcome the destabilizing tendency from the energetic particles. The external source then slowly causes an increase of the slope until the critical slope, as represented by the dashed curve in figure (6), is reached. When the critical slope is barely exceeded, there is a discrete number of separate single mode

oscillations with a very small mode width proportional to $v_{e f f}\left(1-\gamma_{d} / \gamma_{L}\right)^{1 / 4}$. When barely above marginal stability, the mode width is too small to cause overlap. However, the source causes a further increase in the slope, so that the mode width increases. If there are enough modes present, mode overlap occurs, at the slope level shown by the solid curve in figure (6). After mode overlap is triggered, there is an increase in the region of flattening as particles are no longer restricted to diffuse within the environs of a single separatrix, but now can diffuse to neighboring resonances. The increase in the range of flattening of the distribution, then leads to an enhancement the free energy released to the wave, causing an increase of mode amplitude, as was previously discussed in section (3). Hence, a rapid relaxation to a lower slope arises as shown by the dotted curve, well below the critical slope. Then the modes are stabilized, the waves damp away, and the external source then produces an increase of the slope at the classical rate as the cycle repeats itself. If the target energetic particle slope, that would arise in the absence of oscillations, is well above the critical slope, the maximum slope that is experimentally achieved will only be somewhat above the critical slope for instability; well below the desired target slope.

Numerical particle simulations have been performed in [11] that illustrates an amplification of mode amplitude due to mode overlap as shown in figure (7). An initial state is taken where the distribution is assumed to be at its classical targeted slope and then 


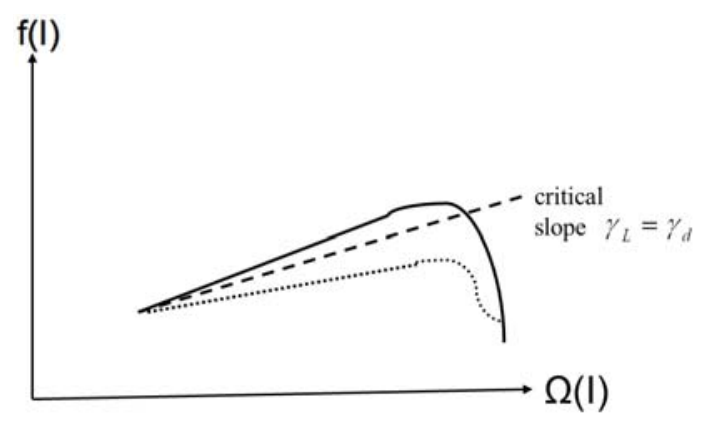

FIGURE 6. Oscillation cycle of marginal stable state. When distribution's slope is below the critical one(dashed curve), the slope builds up at the classical rate, to the critical slope for the onset of instability. First, single non-overlapping modes are excited, allowing overall slope to continue to increase, to where mode overlap arises (solid curve). Then rapid particle loss ensues, lowering slope to well below critical one, causing background dissipation to damp the excited modes whereupon particles source restarts slope buildup to continue oscillation cycle.

the response to spontaneous wave excitation is considered. First consider the first pulse shown in the top left figure, where two modes with relatively close resonant frequencies are present. Initially, the instabilities grow at their linear growth rate $\gamma_{L}$. At a time, $\gamma_{L} t=73$ the mode amplitude is shown on the upper left panel at the time indicated by the left-most of the three arrows. The excited wave energy is relatively low, but we see in the panel on the upper right side that flattening of the spatially averaged distribution function has been achieved in two separate resonant regions of $\Omega$ (called velocity in the figure). However, mode saturation does not occur at this time, as clearly seen by the increase of wave energy at the middle arrow around the time $\gamma_{L} t=85$ as compared to the wave energy at $\gamma_{L} t=73$. At the later time the lower left panel shows an annealing of the distribution between the two resonances but also that the particle mixing is extending over a broader band in $\Omega$, which is due to the combination of particle mixing between the resonance regions due to mode overlap and the increasing mode amplitudes. Because more phase space is available for particles to mix, more free energy is being released and the excited saturation levels can exceed their single mode saturation levels. At the right-most arrow at $\gamma_{L} t=98$ the excited wave energy has reached its peak saturation level at a level dramatically above the level of two single modes alone and the distribution has flattened over a broadened region of $\Omega$ as shown in the lower right panel. As the simulation continues, we see repetitions of the pulse rate, each of which lead to a strong flattening of the distribution followed by a relatively long buildup time for when the slope of the destabilizing distribution to be nearly restored,

We can estimate the level of enhancement in the scaling as follows. For a single resonant mode it can be ascertained that the energy lost in the resonant region is proportional to $\gamma_{L} \omega_{b}^{3}$ while the level of wave energy excitation is proportional to the square of the mode amplitude, or equivalently to $\omega_{b}^{4}$. Thus if on the time scale of the growth rate, the wave damping $\gamma_{d}$ and energy input from sources are sufficiently small, the overall change of normalized energy is given by, $\alpha \omega_{b}^{4}-\beta \gamma_{L} \omega_{b}^{3}$, where $\alpha$ and $\beta$ are normalized proportionality constants. As the overall energy is nearly conserved in a time scale 

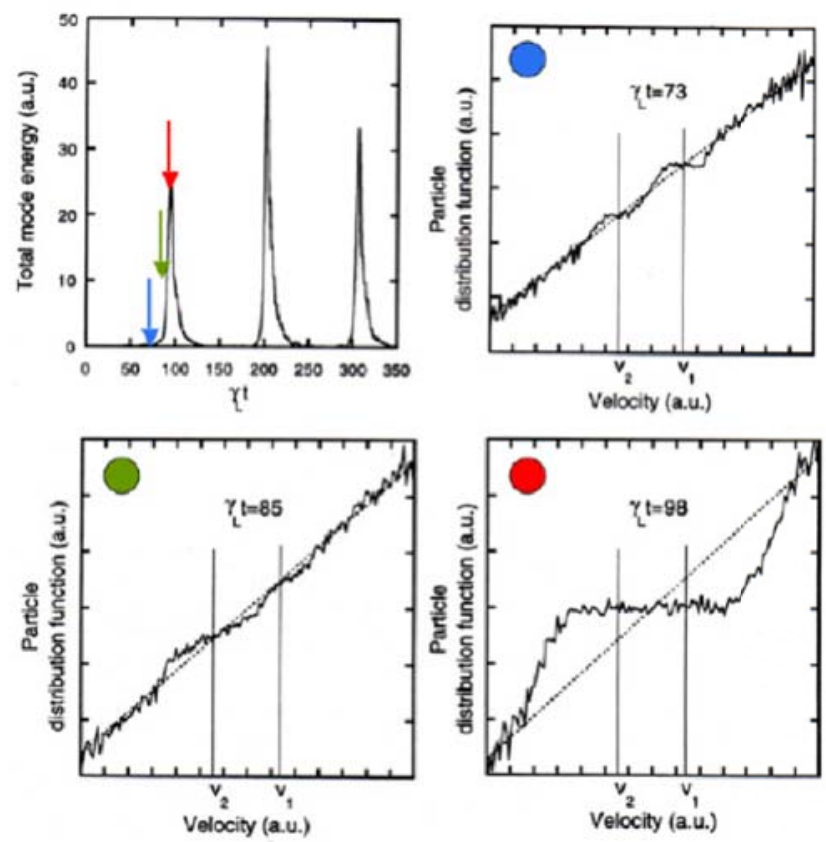

FIGURE 7. Simulation of relaxation cycle due to two closely resonant modes.

$\sim 1 / \gamma_{L}$, we find that the saturation level is given by $\omega_{b} \sim \beta \gamma_{L} / \alpha$, a scaling noted earlier. However, we see in the upper left figure that the wave energy level does not saturate at the time $\gamma_{L} t=73$, but continues to grow. This is because the two waves are too close to each other and particles in one resonant region can mix with particles in the neighboring resonance region giving rise to mode overlap and a further release of energetic particle kinetic energy as a result of the mixing. As the two modes are only assumed to be separated from each other by an amount $\Delta \Omega \approx \gamma_{L}$, the energetic particles mix in this case over a region $\sim 2 \gamma_{L}$, which gives rise to a loss of resonant particle energy that is given by $\gamma_{L}\left(2 \gamma_{L}\right)^{3} \beta$, while the total wave energy is given by $\left(2 \omega_{b}\right)^{4}$. By again applying energy conservation, the expected total saturated wave energy per mode is found to be as large as 4 times the saturated energy level for two well separated modes with the same growth rate. If this argument is further extended to $\mathrm{N}$ roughly equally separately modes with a phase space separation $\Delta \Omega \sim \gamma_{L}$ between each of the modes, the factors of 2 appearing explicitly in the two mode overlap discussion, gets replaced by $\mathrm{N}$, and then the amplification of wave energy released per mode scales by a factor of $N^{2}$ above the single mode case. Clearly, this wave release amplification can be a serious problem that needs to be avoided.

\section{e. Quasilinear theory}

The generic method to treat transport from waves is to employ quasilinear theory. The conventional form of quasilinear equation when cast in terms of the wave trapping 
frequency that we have discussed throughout this paper is,

$$
\frac{\partial f(\vec{J})}{\partial t}-\frac{\pi}{2} \sum_{j, k}\left|\omega_{b j, k}^{4}\right| \frac{\partial}{\partial \Omega_{j, k}} \mathscr{Q}\left(\omega_{k}-\Omega_{j, k}\right) \frac{\partial f(\vec{J})}{\partial \Omega_{j, k}}=S r
$$

where the subscript $k$ refers to a linear eigenmode and the subscript $j$ refers to the specific region in phase space associated with the mode frequency $\omega_{k}$. The right hand side of the quasilinear equation represents additional phase space sources, sinks and collisional relaxation terms that are part of the problem. The quantity $\mathscr{Q}\left(\omega_{k}-\Omega_{j, k}\right)$ is the frequency correlation function, with the normalization property $\int d \Omega_{j, k} \mathscr{Q}\left(\omega_{k}-\Omega_{j, k}\right)=$ 1. The correlation function needs to be a peaked function in phase space. During the linear wave growth of the wave, its analytic form has been derived to be,

$$
\mathscr{Q}\left(\omega_{k}-\Omega_{j, k}\right)=\frac{\gamma_{j, k}}{\pi\left(\left(\omega_{k}-\Omega_{j, k}\right)^{2}+\gamma_{j, k}^{2}\right)} \longrightarrow \delta\left(\omega_{k}-\Omega_{j, k}\right),
$$

in the limit where $\gamma_{j, k}=\gamma_{L j, k}-\gamma_{d j, k}$, the linear growth rate of the mode, is taken to be very small. The delta function form, $\mathscr{Q}\left(\omega_{k}-\Omega_{j, k}\right)=\delta\left(\omega_{k}-\Omega_{j, k}\right)$, is the most frequently chosen form found in the literature. More generally we intuitively interpret the width of the function $\mathscr{Q}\left(\omega_{k}-\Omega_{j, k}\right)$ as the region of mixing of the resonant particles. The minimum width is taken to be comparable to the trapping frequency $\omega_{b j, k}$. In addition the width broadens due to diffusive mixing through the resonance region which adds the width, $v_{e f f}^{3} /\left(\omega_{b j, k}^{2}+\gamma_{j, k}^{2}\right)$, to the mixing region. Hence the intuitive width, $\gamma_{w d j, k}$ of resonant particle in the resonance region is, $\gamma_{w d j, k} \sim a_{j, k} v_{e f f}^{3} /\left(\omega_{b j, k}^{2}+\gamma_{j, k}^{2}\right)+$ $b_{j, k} \gamma_{j, k}+c_{j, k} \omega_{b j, k}$ with the coefficients, (a,b,c) being order unity quantities selected to best fit saturations results found in particle simulation [12]. Thus, a proposed correlation function would have the form of Eq.(20), with the replacement of $\gamma_{j, k}$ by $\gamma_{w d j, k}$. Indeed, the specific functional form of the peaked function $\mathscr{Q}\left(\omega_{k}-\Omega_{j, k}\right)$ is not truly essential as long as the integral unit normalization condition $\int d \Omega_{j, k} \mathscr{Q}\left(\omega_{k}-\Omega_{j, k}\right)=1$ is satisfied. In particular we note that the tail of the Lorenztian form of Eq.(20) is too large since eventually, without any diffusive effects, the response in the tail is adiabatic. Instead it is more appropriate to choose as a form, a single peaked positive unit normalized correlation function $\mathscr{Q}$ as either Gaussian, step function or etc. in the dimensionless variable $\left(\omega_{k}-\Omega_{j, k}\right) / \gamma_{w d j, k}$.

In addition we use the wave equation given by Eq.(13) for the resonant particle response with the current from linear theory substituted into the resonant particle current. A strictly formal perturbation theory gives a result for the wave energy evolution,

$$
\begin{aligned}
& \beta_{j k_{0}}\left(\frac{\partial \omega_{b j, k_{0}}^{4}}{\partial t}+2 \gamma_{d j} \omega_{b j, k_{0}}^{4}\right) \\
& =\frac{n}{\omega_{k}} \frac{\pi}{2} \int d J_{2}^{\prime} d J_{3}\left(\frac{\partial J_{1}\left(\Omega_{j, k}=0\right)}{\partial \Omega_{j, k}}\right)^{2} \mathscr{Q}_{w}\left(\omega_{k}-\Omega_{j, k}\right) \omega_{b j, k}^{4} d \Omega_{j, k} \frac{\partial f}{\partial \Omega_{j, k}}
\end{aligned}
$$

where $\mathscr{Q}_{w j, k}\left(\omega_{k}-\Omega_{j, k}\right)=\delta\left(\omega-\Omega_{j, k}\right)$ and $\beta_{j, k} \omega_{b j, k_{0}}^{4}$ is the wave momentum of the mode in terms of a reference trapping frequency $\omega_{b j, k_{0}}$, for a particular k-value, $k_{0}$. 
However, this form together with the quasilinear equation for the distribution does not lead to conservation of the total momentum of the system. To achieve conservation of total momentum, we change the delta function form of the correlation function to the same form used in the quasilinear equation, ie. $\mathscr{Q}_{w j}\left(\omega_{k}-\Omega_{j, k}\right)=\mathscr{Q}\left(\omega_{k}-\Omega_{j, k}\right)$. This form is still somewhat deficient as it does not lead to exact conservation of the total energy. However total energy is approximately conserved to the extent that $\left(\omega_{k}-\right.$ $\left.\Omega_{j, k}\right) / \gamma_{w d j, k}$ is a peaked function compared to the widths of the ambient distribution function and the eigenfunctions.

This description of quasilinear theory enables its use over all regimes, from the extremes where there is complete mode isolation to where there is complete mode overlap and including the transition regime between the two extreme cases. As previously noted, there is a considerable difference in the amount wave energy released between the isolated case and the overlapped case, with much more energy released when the resonances overlap. This increase of wave energy can even lead to an avalanche. Then a small change of system parameters leads to dramatic differences in the nonlinear state. In one case, the waves hardly change the distribution of energetic particles compared to a totally quiescent case to another case where an additional parameter increment causes nearly all the energetic particles to be lost. In figure (8) we show the results of a bumpon-tail simulation studied in [12]. On the top row we see seven independent modes producing benign oscillations where the stored particle energy is hardly affected by the presence of the mode, as can be inferred from the top two panels in fig. (8). The spacing between the modes was deliberately chosen to produce a 'domino' effect in the system. The modes in the bottom of the upper left figure are more closely packed than the modes in the top of that figure. In the top figures, the inner two modes do not quite overlap. With a slightly increased linear growth rate, the width of the inner most resonance expands just enough to allow overlap of the lower two resonances to produce an increased wave energy of about four times the wave energy released by two non-overlapping modes, which increases the width of the saturated wave, thereby enabling wave overlap with the third mode from the bottom. There then follows an even larger amplification of wave energy. The 'blast' continues upward until all the modes overlap. At that stage there is rapid diffusion over the entire phase space with a considerably enhanced wave amplitudes compared to the non-overlap case. The result is that nearly the entire distribution is lost to left hand particle absorbing boundary, in a time scale of $\sim 1 / \gamma_{L}$.

\section{SUMMARY AND CONCLUSION}

We have described the formalism together with analytic and numerical results for how to estimate the saturation level of discrete waves in a plasma that exists in absence of dissipation or instability drive (e.g. electrostatic plasma waves, shear Alvén-like waves such as the Toroidal Alfvén Eigenmode, etc.) when they are driven unstable by a kinetic particle/wave resonant interaction of an inverted energetic particle distribution. By first developing a nonlinear theory for a single wave with non-overlapping resonances we showed how the conventional quasilinear theory, which generally requires resonance wave overlap for its applicability, can be generalized to incorporate the fully isolated and fully overlapped regimes, as well as the transition regime between the two. Depending 

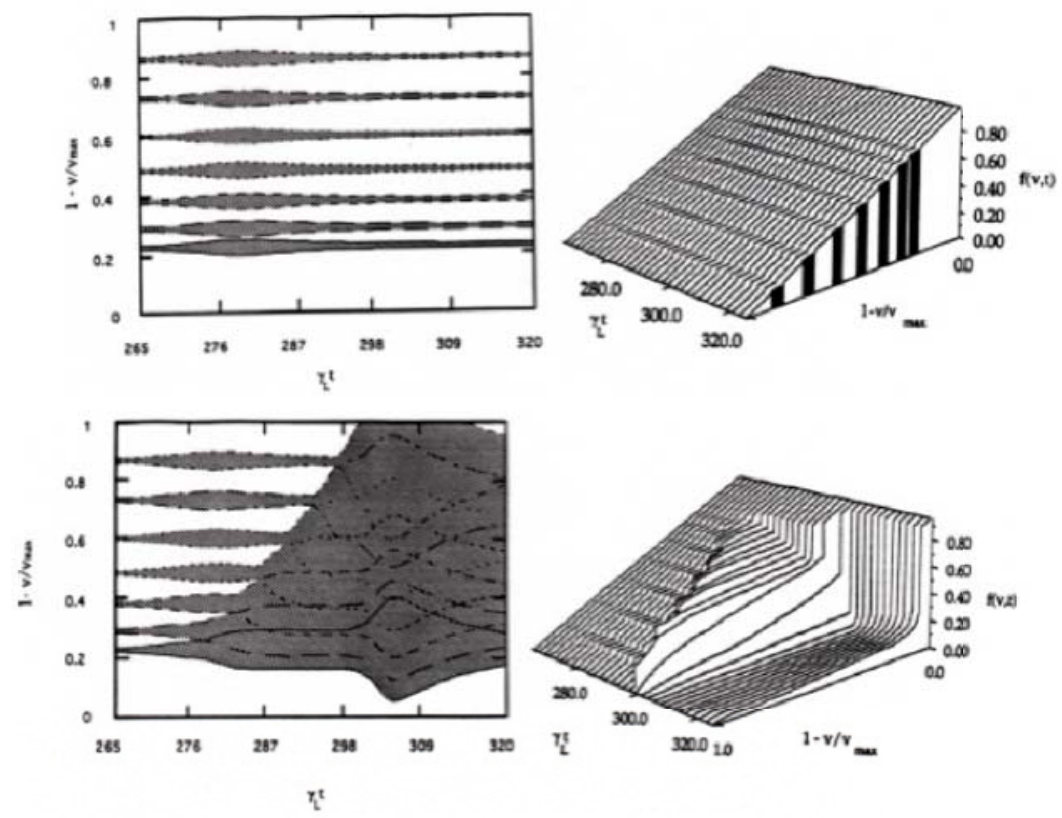

FIGURE 8. Simulation of global avalanche using quasilinear theory with modified resonance widths. See text for detail description.

on parameters, these models produce steady state as well as pulsating responses for the plasma. In addition it is noted that there is a possibility of a phase space avalanche (a catastrophe in the parlance of nonlinear dynamics) being triggered in the system, where a small change of system parameters triggers a transition from a benign state to a state where rapid global diffusion is produced. The author believes that the quasilinear model briefly described in this article will prove to be an invaluable tool for interpreting and predicting the effects of alpha particles in regimes where they cause shear Alfvén-like modes to be excited. A concerted scientific effort should be made to build a suitable quasilinear code that can predict and track global alpha particle dynamics when they excite Alfvénic instability in a fusion producing experiment such as planed for the ITER project.

The work described here has also led to deeper insight into the single mode problem near marginal stablility. Only the steady states results of this study were described in this paper. The study of the non-steady state regimes $\left(v_{e f f} /\left(\gamma_{L}-\gamma_{d}\right)<1\right)$ has lead to the observation of rapid frequency chirping due to the formation of phase space structures $[13,14]$. This theory has been described by M. Lilley at this summer school session and is leading to deep insights into non-linear behavior of alpha particles in a burning plasma. For further reading, with an extensive correlation to experimental phenomena, the reader is referred to the review paper by Breizman and Sharapov [15]. 


\section{REFERENCES}

1. K. Miyamoto, Plasma physics for nuclear fusion, MIT Press, 1980, ISBN 9780262131452.

2. L. Landau, and E. Lifshitz, Mechanics, Course of theoretical physics, Butterworth-Heinemann, 1976, ISBN 9780750628969.

3. T. O'Neil, Physics of Fluids 8, 2255-2262 (1965).

4. H. L. Berk, and B. N. Breizman, Physics of Fluids B 2, 2235-2245 (1990).

5. B. Fried, C. Liu, R. Means, R. Sagdeev, and C. U. L. A. P. P. GROUP., Nonlinear Evolution and Saturation of an Unstable Electrostatic Wave, Defense Technical Information Center, 1971.

6. B. N. Breizman, H. L. Berk, M. S. Pekker, F. Porcelli, G. V. Stupakov, and K. L. Wong, Physics of Plasmas 4, 1559-1568 (1997).

7. N. N. Gorelenkov, Y. Chen, R. B. White, and H. L. Berk, Physics of Plasmas 6, 629-632 (1999).

8. N. V. Petviashvili, Coherent Structures in Nonlinear Plasma Dynamics, PhD dissertation, University of Texas at Austin, Department of Physics (1998).

9. H. L. Berk, B. N. Breizman, and H. Ye, Physical Review Letters 68, 3563-3566 (1992).

10. H. L. Berk, B. N. Breizman, and N. V. Petviashvili, Physics Letters A 234, 213-218 (1997).

11. H. L. Berk, B. N. Breizman, and M. Pekker, Physics of Plasmas 2, 3007-3016 (1995).

12. H. L. Berk, B. N. Breizman, J. Fitzpatrick, and H. V. Wong, Nuclear Fusion 35, 1661-1668 (1995).

13. H. L. Berk, B. N. Breizman, and M. Pekker, Physical Review Letters 76, 1256-1259 (1996).

14. M. K. Lilley, B. N. Breizman, and S. E. Sharapov, Physical Review Letters 102, 195003 (2009).

15. B. N. Breizman, and S. E. Sharapov, Plasma Physics and Controlled Fusion 53, 054001 (2011). 